$\xi=-$ 国

\title{
Face Recognition Using Location Averaging and Intensity's Position Estimation Techniques for Human Authentication
}

\author{
Parivazhagan. $\mathrm{A}^{1 *}$, Dr.Brintha Therese. $\mathrm{A}^{2}$ \\ ${ }^{1 *}$ PhD Fulltime Research Scholar, School of Electronics Engineering, VIT University, Chennai Campus, India \\ ${ }^{2}$ Professor, School of Electronics Engineering, VIT University, Chennai Campus, India \\ *Corresponding author E-mail: parivazhagan.a2013@vit.ac.in,parivazhaganphd@gmail.com
}

\begin{abstract}
Face recognition is an effective tool in the biometric human recognition system. In this competitive world, several techniques and systems are emerging to satisfy the needs of the face recognition system's performance. To obtain the high-performance ratio novel techniques are combined and created a new face recognition system. Spatial domain techniques like Gray averaging technique, Location averaging technique and Intensity's position estimation technique are united with frequency domain technique like Discrete Cosine Transform. Intensity's position estimation is a novel feature extraction and classification technique proposed in this work. Three standard face databases are tested using this system. Accuracy and runtime are major parameters used to validate the obtained results. The maximum accuracy rate of about $86 \%$ is obtained.
\end{abstract}

Keywords: Biometrics, Face recognition, Human recognition system, Gray averaging, Location averaging, Intensity's position estimation

\section{Introduction}

Face recognition $[2,3]$ plays a major role in human authentication for security issues $[4,6]$. Several novel techniques are arising every day to solve the errors in face recognition system $[7,8$, and 9]. This work deals with pose variation problem in face images and to solve it techniques are proposed. This face recognition system consists of the test image and database images both are same in size and different in the pose. This system flows through Gray averaging technique, Location averaging technique, Discrete cosine transform and Intensity's position estimation.

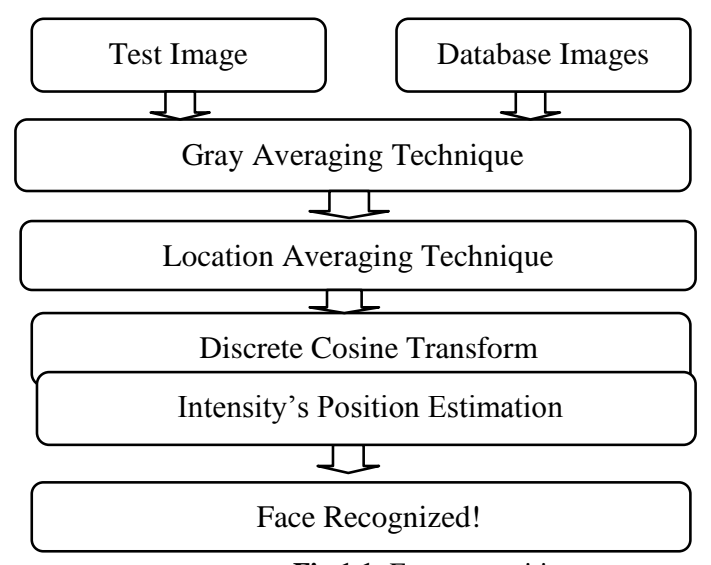

Fig 1.1: Face recognition

Three different set of standard face databases are used in this Face recognition system and the overall process is shown in Fig.1.1. The rest of this paper is organized as 2.Gray averaging technique, 3.Location averaging technique, 4.Discrete cosine transform,
5.Intensity's position estimation, 6.Results and discussions, 7.Conclusions and References.

\section{Gray Averaging Technique}

This technique is applied to find the difference between maximum gray scale value (256) and image intensity value. Hence it is representing the difference, between the model image $\mathrm{x}$ and $\mathrm{d}(\mathrm{d}=256$ it denotes 256 shades of gray), and it gives the result as y. Then the differences of values are found between the $\mathrm{x}$ and $\mathrm{y}$ according to the Eq. 2.1.The final result is denoted as S. A model example of Gray - Averaging technique is shown in Fig.2.1. The results of this technique are called as Gray averaged database images and Gray averaged test image.

$y=256-x ; d=256 ; x=$ image

$$
\mathrm{S}=\sqrt{(x-y)^{2}} / \mathrm{d}
$$

\begin{tabular}{|c|c|c|}
\hline 25 & 23 & 72 \\
\hline 45 & 12 & 120 \\
\hline 100 & 200 & 90 \\
\hline 231 & 233 & 184 \\
\hline 211 & 244 & 136 \\
\hline 156 & 56 & 166 \\
\hline 0.8047 & 0.8203 & 0.4375 \\
\hline 0.6484 & 0.9063 & 0.0625 \\
\hline 0.2188 & 0.5625 & 0.2969 \\
\hline
\end{tabular}

Fig 2.1: Gray - Averaging technique 


\section{Location Averaging Technique}

The location averaging technique [5] is an averaging process that transforms an image into its column vector size. It is useful for feature reduction and recognition. The cell elements $\left(\mathrm{C}_{\mathrm{kt}}\right)$ (intensity values) of the image and its corresponding location values are multiplied together, and then divided by its column size $\left(\mathrm{C}_{\mathrm{s}}\right)$, hence it results in the Location averaged value of the Cell $\left(\mathrm{X}_{\mathrm{kt}}\right)$. Location averaged value of the Cell $\left(\mathrm{X}_{\mathrm{kt}}\right)(\mathrm{Eq} .3 .1)$ is found for all the elements in an image after that add all the $X_{\mathrm{kt}}$ present in each row and divide it by the column size $\left(\mathrm{C}_{\mathrm{s}}\right)$.It results in Location averaged value of the row $\left(\mathrm{Y}_{\mathrm{k}}\right)$ (Eq.3.2). For example in an image, the first row elements are transformed into single element called location averaged value of the first row $\left(\mathrm{Y}_{1}\right)$ and this procedure is similarly followed for all the rows. At the end all the row's Location averaged values are collected in a single column vector. Hence, an image is transformed into the vector. A model image's matrix is tested using Location averaging technique and is shown in Fig 3.1 .

$$
\begin{aligned}
& \mathrm{X}_{\mathrm{kt}}=\left(\mathrm{C}_{\mathrm{kt}} * \mathrm{~L}_{\mathrm{kt}}\right) / \mathrm{C}_{\mathrm{s}} \quad \ldots \text { (Eq.3.1) } \\
& \mathrm{Y}_{\mathrm{k}}=\left(\mathrm{X}_{\mathrm{k} 1}+\mathrm{X}_{\mathrm{k} 2}+\mathrm{X}_{\mathrm{k} 3}+\ldots+\mathrm{X}_{\mathrm{kN}}\right) / \mathrm{C}_{\mathrm{s}} \ldots(\text { Eq.3.2) } \\
& \mathrm{Y}_{(\mathrm{k}=1)}=\left(\mathrm{X}_{11}+\mathrm{X}_{12}+\mathrm{X}_{13}+\ldots+\mathrm{X}_{1 \mathrm{~N}}\right) / \mathrm{C}_{\mathrm{s}} \quad ; \quad \text { Here } \mathrm{k}=1 ; \mathrm{t}=1 \text { to } \mathrm{N}
\end{aligned}
$$

$\left[\mathrm{Y}_{1}\right.$

Location averaged value for ' $\mathrm{N}$ ' Rows $=$...

$\left.\mathrm{Y}_{\mathrm{N}}\right]$

$\mathrm{C}_{\mathrm{kt}}-$ Cell value (Intensity value)

$\mathrm{L}_{\mathrm{kt}}-$ Cell Location value; $\mathrm{C}_{\mathrm{s}}-$ Column Size

$\mathrm{k}$ - Row; $\mathrm{t}$ - Column; $\mathrm{N}$ - Last element

$\mathrm{X}_{\mathrm{kt}}$ - Location averaged value of the Cell

$\mathrm{Y}_{\mathrm{k}}$ - Location averaged value of the Row

$Y_{1}$-Location averaged value of the First Row;

$\mathrm{Y}_{\mathrm{N}}$ - Location averaged value of the ' $\mathrm{N}^{\text {th }}$, Row

The Gray averaging results are given as input to the Location averaging technique and its results are called as Location averaged database images and Location averaged test image.

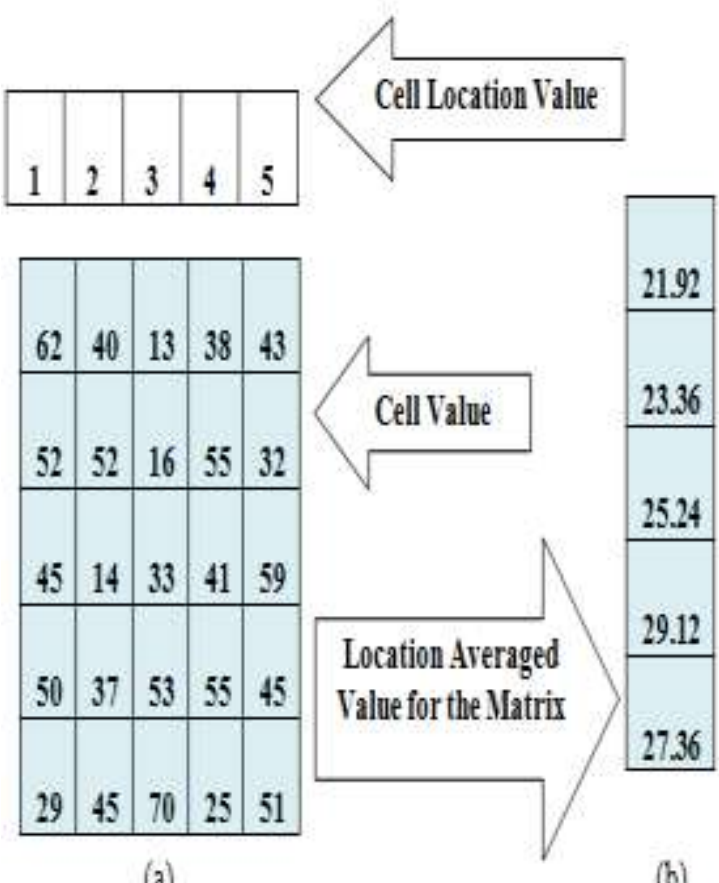

(a)

\section{Discrete Cosine Transform (DCT)}

The cosine based basic functions [1] are applied on the Location averaged test and database images. DCT (Eq.4.1) transforms a signal to frequency domain from its original spatial domain representation. For images, most of the energy values are dominated in the lower frequency spectrum, so while transforming the image, higher frequency coefficients are not given priority and the lower frequency coefficients are taken into account. So here the amount of data needs to describe is reduced, without sacrificing the quality.

$\mathrm{Y}_{\mathrm{m}, \mathrm{n}}=\sum_{i=0}^{N-1} \sum_{j=0}^{N-1} f_{\mathrm{i}, \mathrm{j}} \cos \frac{(2 \mathrm{i}+1) \mathrm{m} \Pi}{2 \mathrm{~N}} \cos \frac{(2 \mathrm{j}+1) \mathrm{n} \Pi}{2 \mathrm{~N}} ; \ldots$

$0 \leq \mathrm{m} \leq \mathrm{N}-1 ; 0 \leq \mathrm{n} \leq \mathrm{N}-1$

The cosine based functions results are named as Location averaged cosine database images and Location averaged cosine test image.

\section{Intensity's Position Estimation}

Here the intensity values of the images are sorted by descending and ascending order, the positions of (Location) the descending and ascending order intensity values are noted. The positions of (Location) sorted, descending and ascending order intensity values of the images are compared for recognition. A model demonstration of Intensity's position estimation technique is shown in Fig.5.1.It contains model Database images (Fig.5.1 (a)) and model Test image (Fig.5.1 (b)). The comparison takes place between the images ascending order intensity's position values (Fig 5.1 (f) (h)) and between the images descending order intensity's position values (Fig 5.1 (d) (g)). While comparing the two images position values similarity must be found (Fig 5.1 (i) (j)). If both the images position values are same it is marked as ' 1 'if not it is marked as ' 0 '. Then the number of one's is summed and the maximum summed value gives the correct matching. From the Model demonstration, shown in Fig.5.1, the maximum summed value is ' 3 '. It shows that the model test image (Fig.5.1 (b)) is matched with the $1^{\text {st }} 1 \mathrm{D}$ vector i.e. $1^{\text {st }}$ image of the model database images (Fig.5.1 (a)). The Intensity's position estimation technique is applied to the Location averaged cosine database images and Location averaged cosine test image. The maximum summed values obtained between the Location averaged cosine database images give the correct matching of database image with the test image.

\section{Results and Discussions}

This Face recognition system is tested on three standard face databases they are, Libor Spacek's face database (150 Subjects) [10], Georgia tech face database (50 Subjects) [11] and VIT RA database (15 Subjects - VIT University, Chennai Campus, Ph.D. scholars face images collected in my Research cabin). The Face recognition results are shown in Fig .6.1 and Table 6.1. The Table 6.1 explains that the maximum Accuracy of about $86 \%$ is achieved for Georgia tech face database even though it has bigger size image. The minimum Average runtime is about 1.058113 sec achieved for VIT RA face database; Libor Spacek's face database yields $83.33 \%$ accuracy in $2.438268 \mathrm{sec}$ Average runtime. The maximum Average runtime of about $4.451159 \mathrm{sec}$ is produced for Georgia tech face database. From the obtained values it is clear that the size of the image is not affecting the accuracy but affecting the runtime. So the larger size images also give perfect accuracy. This system can work with all sizes of images, and the image sizes of the databases are mentioned in Table 6.1. 


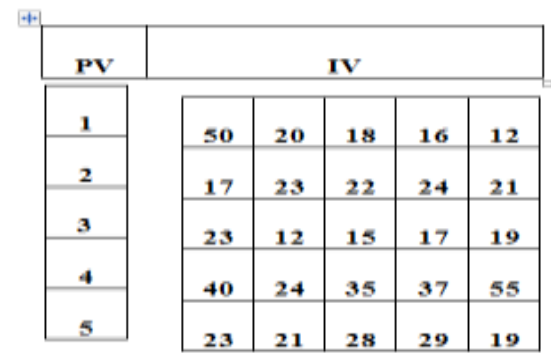

(a) $1 \mathrm{D}$ vectors of 5 images (model Database images) PV - Position value; rV - Intensity value

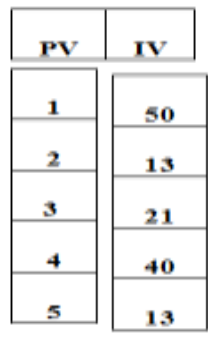

(b) $1 \mathrm{D}$ vector of an image (model Test image) PV - Position value; $\mathrm{rV}$ - Intensity value

\begin{tabular}{|c|c|c|c|c|c|c|c|c|c|}
\hline IV & PV & IV & PV & IV & PV & IV & PV & IV & PV \\
\hline 50 & 1 & 24 & 4 & 35 & 4 & 37 & 4 & 55 & 4 \\
\hline 40 & 4 & 23 & 2 & 28 & 5 & 29 & 5 & 21 & 2 \\
\hline 23 & 3 & 21 & 5 & 22 & 2 & 24 & 2 & 19 & 3 \\
\hline 23 & 5 & 20 & 1 & 18 & 1 & 17 & 3 & 19 & 5 \\
\hline 17 & 2 & 12 & 3 & 15 & 3 & 16 & 1 & 12 & 1 \\
\hline
\end{tabular}

(c) 5 images Intensity values arranged in descending order

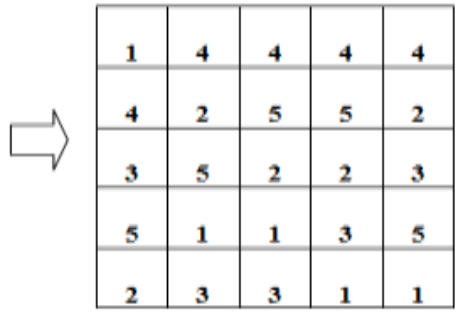

(d) 5 images Descending order Intensity's position values

\begin{tabular}{|c|c|c|c|c|c|c|c|c|c|}
\hline IV & PV & IV & PV & IV & PV & IV & PV & IV & PV \\
\hline 17 & 2 & 12 & 3 & 15 & 3 & 16 & 1 & 12 & 1 \\
\hline 23 & 3 & 20 & 1 & 18 & 1 & 17 & 3 & 19 & 5 \\
\hline 23 & 5 & 21 & 5 & 22 & 2 & 24 & 2 & 19 & 3 \\
\hline 40 & 4 & 23 & 2 & 28 & 5 & 29 & 5 & 21 & 2 \\
\hline 50 & 1 & 24 & 4 & 35 & 4 & 37 & 4 & 55 & 4 \\
\hline
\end{tabular}

(e) 5 images Intensity values arranged in ascending order

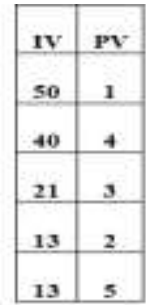

(g) An image'" Intensity values arranged in Dencending order

\begin{tabular}{|l|l|l|l|l|}
\hline 1 & 0 & 0 & 0 & 0 \\
\hline 1 & 0 & 0 & 0 & 0 \\
\hline 1 & 0 & 0 & 0 & 1 \\
\hline 0 & 0 & 0 & 0 & 0 \\
\hline 0 & 0 & 0 & 0 & 0 \\
\hline
\end{tabular}

\begin{tabular}{|l|l|l|l|l|}
\hline & & & & \\
3 & 0 & 0 & 0 & 1 \\
\hline
\end{tabular}

(i) Descending order intensity's position value comparison results

\begin{tabular}{|l|l|l|l|l|}
\hline 2 & 3 & 3 & 1 & 1 \\
\hline 3 & 1 & 1 & 3 & 3 \\
\hline 5 & 5 & 2 & 2 & 5 \\
\hline 4 & 2 & 5 & 5 & 2 \\
\hline 1 & 4 & 4 & 4 & 4 \\
\hline
\end{tabular}

(f) 5 images Ascending order intensity's Position values

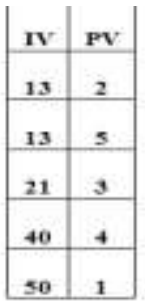

(b) An image' $x$ Intensity values arranged in Ascvnding otder

\begin{tabular}{|l|l|l|l|l|}
\hline 1 & 0 & 0 & 0 & 0 \\
\hline 0 & 0 & 0 & 0 & 0 \\
\hline 0 & 0 & 0 & 0 & 0 \\
\hline 1 & 0 & 0 & 0 & 0 \\
\hline 1 & 0 & 0 & 0 & 0 \\
\hline 3 & 0 & 0 & 0 & 0 \\
\hline
\end{tabular}

(j) Ascending order intensity's position value comparison results
Maximum

Summed Value is ' 3 '

Fig.5.1: Intensity’s position estimation technique 


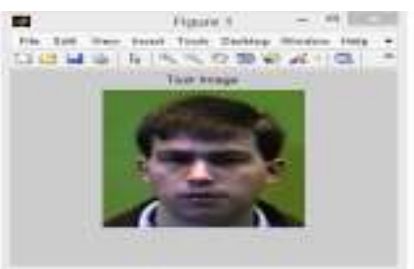

(a)Test Image

Fig 6.1.1: Libor Spacek's face database face recognition results
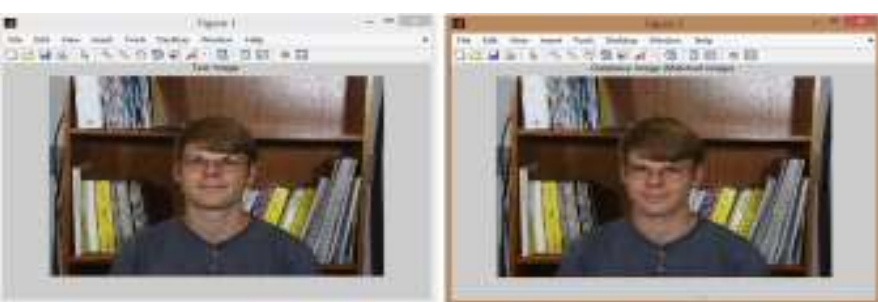

(a)Test Image

Fig 6.1.2: Georgia tech face database face recognition results
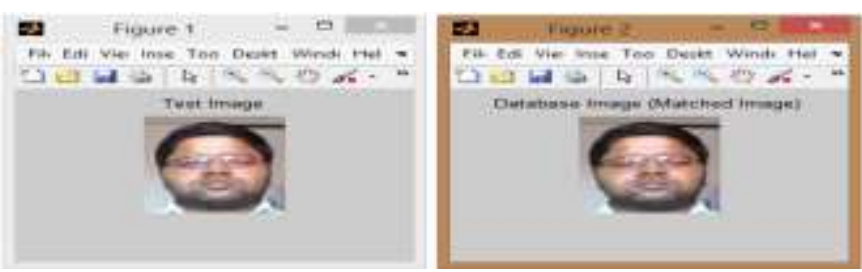

(a)Test Image (b) Database Image (Matched Image)

Fig 6.1.3: VIT RA face database face recognition results Fig 6.1: Face Recognition Results

Table 6.1: Face Recognition Results

\begin{tabular}{|c|c|c|c|c|}
\hline \multirow[b]{2}{*}{$\begin{array}{l}\text { S. } \\
\text { No }\end{array}$} & \multirow[t]{2}{*}{ Parameter } & \multicolumn{3}{|c|}{ Database } \\
\hline & & $\begin{array}{l}\text { Libor } \\
\text { Spacek's } \\
\text { face data- } \\
\text { base } \\
\text { (150 } \\
\text { Subjects) }\end{array}$ & $\begin{array}{l}\text { Georgia } \\
\text { tech face } \\
\text { database } \\
\text { (50 } \\
\text { Subjects) }\end{array}$ & $\begin{array}{l}\text { VIT RA } \\
\text { Face data- } \\
\text { base } \\
\text { (15 } \\
\text { Subjects) }\end{array}$ \\
\hline 1 & Input Image size & $180 \times 200$ & $640 X 480$ & $90 \times 110$ \\
\hline 2 & $\begin{array}{l}\text { Face Recognized } \\
\text { (Accuracy) }(\%)\end{array}$ & 83.33 & 86 & 73.33 \\
\hline 3 & $\begin{array}{l}\text { Face Not } \\
\text { Recognized }(\%)\end{array}$ & 16.66 & 14 & 26.66 \\
\hline 4 & $\begin{array}{l}\text { Average Runtime } \\
\text { (Sec) }\end{array}$ & 2.438268 & 4.451159 & 1.058113 \\
\hline
\end{tabular}

\section{Conclusions}

This proposed work is concentrated on the pose variation problems of the Face images. The same size images are compared together for perfect matching. The three different face databases have different pose varied face images and it gives effective results. The maximum accuracies of about $86 \%$ and $83.33 \%$ are achieved with the minimum average runtime. The larger size and smaller size images are tested using this system and it proved that this system can work for all sizes of images.

\section{References}

[1] Weilong Chen, Meng Joo Er, and Shiqian Wu, Illumination Compensation and Normalization for Robust Face Recognition Using Discrete Cosine Transform in Logarithm Domain, IEEE Transactions on Systems, Man, and Cybernetics-Part B: Cybernetics, Vol. 36, No. 2, April 2006, Pp 458-466.

[2] Somaye Ahmadkhani, Peyman Adibi, Face recognition using supervised probabilistic principal component analysis mixture model in dimensionality reduction without loss framework, IET Computer Vision, 2016, Vol. 10, Issue 3, pp. 193-201.

[3] Vinay. A, Vinay S. Shekhar, Akshay Kumar. C, Natarajan. S, K.N. Balasubramanya Murthy, Affine-scale invariant feature transform and two-dimensional principal component analysis: a novel framework for affine and scale invariant face recognition, IET Computer Vision, 2016, Vol. 10, Issue 1, pp. 43-59.

[4] Zahid Mahmood, Tauseef Ali, Samee U. Khan, Effects of pose and image resolution on automatic face recognition, IET Biometrics, 2016, Vol. 5, Issue 2, pp. 111-119.

[5] Parivazhagan A and BrinthaTherese A. (2018) Combined Analysis of Image Processing Transforms with Location Averaging Technique for Facial and Ear Recognition System, Computational Signal Processing and Analysis, Lecture Notes in Electrical Engineering, Volume 490, Springer, Singapore, pp 67-77.

[6] Parivazhagan A., BrinthaTherese A, A Novel 2D Face, Ear Recognition System Using Max-Min Comparison Technique for Human Identification, Artificial Intelligence and Evolutionary Computations in Engineering Systems, Advances in Intelligent Systems and Computing (AISC), Vol 517, Springer, Singapore, July 2017, pp 695-704.

[7] Chunlei Peng, Xinbo Gao, Nannan Wang, and Jie Li, Graphical Representation for Heterogeneous Face Recognition, IEEE Transactions on Pattern Analysis and Machine Intelligence, Vol. 39, No. 2, February 2017, pp. 301-312.

[8] June-Young Jung, Seung-Wook Kim, Cheol-Hwan Yoo, Won-Jae Park, and Sung-Jea Ko, LBP-Ferns-Based Feature Extraction for Robust Facial Recognition, IEEE Transactions on Consumer Electronics, Vol. 62, No. 4, November 2016, pp. 446-453.

[9] Srinivasa Perumal Ramalingam, P.V.S.S.R. Chandra Mouli, Twolevel dimensionality reduced local directional pattern for face recognition, International Journal Biometrics, Vol. 8, No. 1, 2016, pp.52-64.

[10] Libor Spacek's Facial Images Databases: http://cmp.felk.cvut.cz/ spacelib/faces/ (URL).

[11] Georgia Tech Face Database, http://www.anefian.com/research/face_reco.htm (URL) 